\title{
ARTICLE
}

\section{Field-deployable rapid multiple biosensing system for detection of chemical and biological warfare agents}

Masato Saito ${ }^{1,2}$, Natsuko Uchida ${ }^{3}$, Shunsuke Furutani ${ }^{2,4}$, Mizuho Murahashi ${ }^{1}$, Wilfred Espulgar ${ }^{1}$, Naoki Nagatani ${ }^{5}$, Hidenori Nagai ${ }^{2,4}$, Yuki Inoue ${ }^{1}$, Tomohiko Ikeuchi ${ }^{1}$, Satoshi Kondo ${ }^{3}$, Hirotaka Uzawa ${ }^{3}$, Yasuo Seto ${ }^{6}$ and Eiichi Tamiya ${ }^{1}$

The threat of biological and chemical terror acts remains a growing worldwide concern. There is therefore a need to develop appropriate technology for the detection of chemical and biological warfare agents, with early identification intended for use by first responders. Here, we disclose the developed autonomous air sampling and detection system for evaluation of the presence of chemical and biological warfare agents that can be harmful to the population. The current device utilizes the designed mist generator-assisted air collection system $\left(3381 \mathrm{~min}^{-1}\right.$ ) and biosensing chip technologies, such as electrochemical measurement, Au nanoparticle-based localized surface plasmon resonance, and rapid microfluidic chip PCR for detection of minute concentrations lower than the mean lethal dose $\left(\mathrm{LD}_{50}\right)$ of nerve gases (sarin and VX), toxic proteins (BTX/A/Hc and ricin), and pathogens (anthrax simulant). An operation time of only 5-15 min is needed for the collection and detection; sample preparation is already integrated into the system without the need for direct human intervention. In addition to the system's sensitivity and ease of use, its portability makes it highly beneficial for first responders, which could aid in immediate risk assessment and mitigation of on-site events.

Keywords: biological and chemical agents; biosensing; electrochemical detection; LSPR; PCR; rapid detection

Microsystems \& Nanoengineering (2018) 4, 17083; doi:10.1038/micronano.2017.83; Published online: 29 January 2018

\section{INTRODUCTION}

In 1995, the nerve gas sarin (O-isopropyl methylphosphonofluoridate) was used as a chemical weapon in a terrorist attack in Tokyo's subway ${ }^{1,2}$. In 2001 and 2004, anthrax attacks and ricin incidents occurred in the USA. In recent years, the lack of reports on successfully executed biological and chemical (BC) terror attacks does not indicate a guarantee of absolute safety. The US Department of State (DOS) reported in 2015 that some countries still fail to comply with the disarmament treaty under the Biological Weapons Convention or Biological and Toxin Weapons Convention and continue to conduct biological weapon-specific activities $^{3}$. This makes the threat of BC terror attacks real and actually growing. Considering the threat and how accessible, cheap, and easy to prepare these BC weapons are, it would be of great significance to develop a rapid, reliable, and highly sensitive detection system for BC warfare agents for on-site use by first responders.

Among many BC agents, sarin, VX (S-[2-(diisopropylamino) ethyl]-O-ethyl methylphosphonothioate), anthrax, botulinum toxin, and ricin have strong acute toxicity and are ranked as 'category A or B' by the Centers for Disease Control and Prevention (USA) and Schedule I compounds according to the Chemical Weapon Convention. Close attention is therefore given to these $B C$ warfare agents with regard to risk management and counter-measures against terrorism.

For the detection of BC warfare agents ${ }^{4-7}$, many methods have been developed. For nerve gas detection, such as sarin, tabun, soman, and VX, mass analyses including GC-MS, LC-MS, IM-TOFMS or MALDI-TOF MS, acetylcholinesterase (AChE) reactivation, colorimetric detection, chromogenic and fluorescent probing based on fluorescence resonance energy transfer, and electrochemical detection have been reported. For anthrax detection, multiplexed immunoassay ${ }^{8}$, PCR using real-time TaqMan assays ${ }^{9}$, and a portable Raman system based on the surface-enhanced Raman scattering using an AgFON substrate ${ }^{10}$ have been utilized. For detection of biological warfare agents, immunochemical methods have been widely used ${ }^{11}$. Recently, autonomous detection of aerosolized biological agents by multiplexed immunoassay with PCR has been developed, in which Bacillus anthracis and Yersinia pestis are detected by a sequence of aerosol sample collection, multiplexed immunoassay analysis, DNA extraction, and confirmatory $\mathrm{PCR}^{9}$. However, this autonomous system is not compatible with other chemical warfare agents' detection. In addition, the conventional technologies reported have shortcomings for first responders' utility, such as concerns of low sensitivity, high false-positive rate, tedious operation, and time-consuming processing. In particular, first responders, such as

\footnotetext{
${ }^{1}$ Department of Applied Physics, Graduate School of Engineering, Osaka University, 2-1, Yamadaoka, Suita, Osaka 565-0871, Japan; ${ }^{2}$ Advanced Photonics and Biosensing Open Innovation Laboratory (PhotoBIO-OIL), National Institute of Advanced Industrial Science and Technology (AIST), Photonics Center Osaka University P3 BIdg, 2-1 Yamada-Oka, Suita, Osaka 565-0871, Japan; ${ }^{3}$ Nanomaterials Research Institute, National Institute of Advanced Industrial Science and Technology (AIST), 1-1-1 Higashi, Tsukuba 305-8565, Japan; ${ }^{4}$ Health Research Institute, National Institute of Advanced Industrial Science and Technology (AIST), 1-8-31 Midorigaoka, Ikeda, Osaka 563-8577, Japan; ${ }^{5}$ Department of Applied Chemistry, Graduate School of Engineering, Okayama University of Science, 1-1, Ridai-cho, Kita-ku, Okayama-shi 700-0005, Japan and ${ }^{6}$ National Research Institute of Police Science, 6-3-1 Kashiwanoha, Kashiwa, Chiba 277-0882, Japan
}

Correspondence: Masato Saito or Hirotaka Uzawa (saitomasato@ap.eng.osaka-u.ac.jp or h.uzawa@aist.go.jp)

Received: 3 April 2017; revised: 5 September 2017; accepted: 13 September 2017 
police mobile teams and fire fighters, require mobile and robust lightweight systems for easy transport and installation for on-site detection, which is crucial for immediate risk assessment, mitigation, and decision-making. Therefore, the development of an autonomous system that can accurately and quickly detect BC warfare agents from sampling at the event site for initial screening is strongly desired.

We have studied and developed a variety of biosensors that accommodate the detection of chemical warfare agents, biological toxins, and pathogens. Chemical warfare agents, such as sarin and VX, inhibit the activity of AChE, which has an important role in the central nervous system ${ }^{12,13}$. AChE activity and its inhibition can be measured by analytical methods, such as spectrophotometric, fluorometric, piezoelectric, potentiometric, and amperometric measurements ${ }^{14-18}$. Electrochemical measurement is desired due to its highly sensitive detection and, more importantly, the portability and miniaturization of capable devices. We have already developed a USB-powered portable potentiostat (BDTminiSTAT100) and a disposable screen-printed carbon electrode (SPCE) with exemplary performance ${ }^{19-21}$. The optimized electrochemical measurements from our devices were selected and then utilized in the developed autonomous system.

Localized surface plasmon resonance (LSPR) technology is a useful sensing method for interaction analysis of biomolecules. The technique is widely applied for immunochemical reaction analysis and for clinical diagnosis of infectious diseases. The portability and simplicity of the LSPR system make it desirable as an integral part of the system. In our previous paper, we reported a highly sensitive detection method of biological toxins using the LSPR sensing method based on glyco-nanotechnology ${ }^{22}$. We demonstrated that the LSPR system enabled us to detect ricin at $30 \mathrm{ng} \mathrm{mL}^{-1}$, Shiga toxin at $10 \mathrm{ng} \mathrm{mL}^{-1}$, and the cholera toxin at $20 \mathrm{ng} \mathrm{mL}^{-1}$. Thus, this system is expected to be applicable for other targeted biological toxins.

For detection of pathogenic agents, PCR is greatly attractive because of its sensitivity and specificity, which allow specific sequences of DNA molecules to be amplified. We have already reported an on-chip PCR technology based on microfluidics

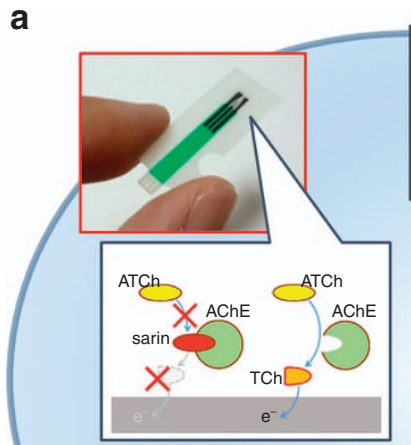

Electrochemical biosensor for nerve gases

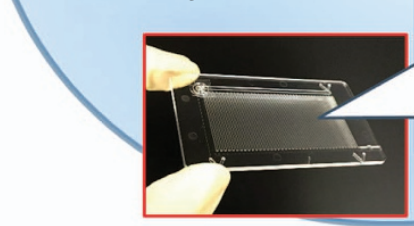

Rapid PCR biosensor for pathogen

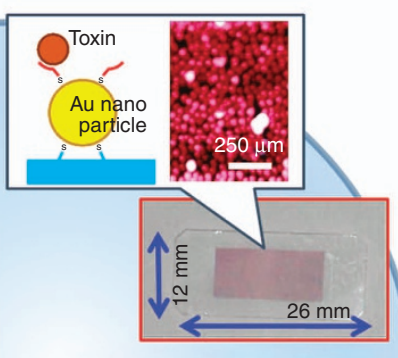

LSPR/glycolipids biosensor for biological toxins
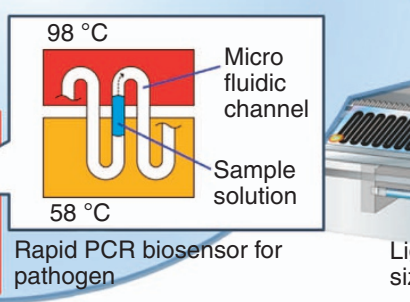

size system

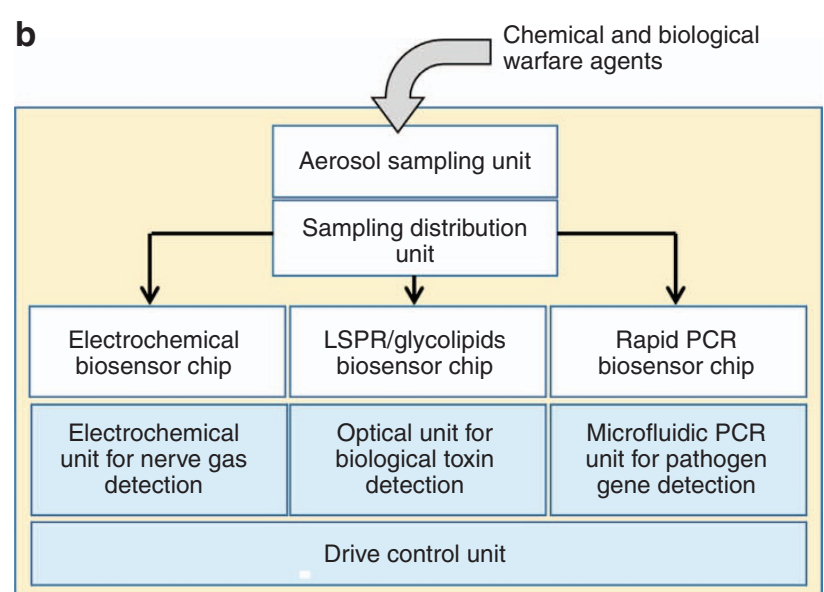

Figure 1 (a) Scheme of our concept of the on-site device system for evaluating the presence of chemical and biological warfare agents rapidly and with high sensitivity. This system works autonomously from air sampling to detection by integrating the air-sampling unit based on cyclone technology and detection system units using biosensor chip device technologies, such as electrochemical measurement, LSPR, and on-chip PCR. In addition, the system is lightweight and compact in size for portability. (b) System composition. Initially, chemical and biological warfare agents in the air are harvested into the aerosol-sampling unit. The collected sample solution is distributed to separated biosensor chips and then measured. All units are operated by the control unit. Note that the parts indicated in the white-colored boxes are considered disposable. ATCh, acetylthiocholine; AChE, acetylcholinesterase; LSPR, localized surface plasmon resonance; TCh, thiocoline. 
technology. We successfully demonstrated the detection of the Anthrax PA gene within $5 \mathrm{~min}$ and influenza virus gene within 10 min, which are faster than by conventional methods ${ }^{19,23}$. Thus, we chose this PCR chip technology and incorporated it into the autonomous system for rapid on-site detection of pathogenic agents.

Moreover, a sample collection system was developed, which is essential for pretreatment and preparation of the target. Biocapture650 (MesoSystems Technology, Inc., Kennewick, USA) is well known as an on-site airborne sampler; however, it has difficulty linking with other devices. In our developed autonomous system, the aerosol formation and the sampling technology were combined, which can aid in easy sample delivery to the detection regions. We developed a unique air-sampling unit that can connect with our biosensors described above.

In summary, in realizing our concept as shown in Figure 1, we integrated our reported biosensing technologies into one system to address various types of agents. We applied the electrochemical measurement with the enzyme reaction and disposable electrode to the nerve gas, the LSPR assay with the glycochip to toxic proteins, and the rapid gene detection with chip PCR to pathogens to detect amounts lower than the mean lethal dosage $\left(\mathrm{LD}_{50}\right)$. Additionally, we have designed and fabricated a unique air-sampling unit for autonomous collection and sampling. This technology not only simply integrates our micro- and nanobiosensing chip technologies but also creates a complete automated system from collection to detection, which are usually done separately in other sensing devices, with manual handling of reagents. Thus, the assembled device does not require a very technically adept operator to be used. In addition, the assembled device is designed for easy handling and transportation. We believe that the assembled device would greatly benefit first responders and aid in immediate evaluation of the risk of on-site events. Here, we present the integrated and automated collection and detection system for the simultaneous recognition of sarin, VX, anthrax, botulinum toxin, and ricin.

\section{MATERIALS AND METHODS \\ General information}

All chemicals were received from commercial suppliers and used without further purification. AChE (human recombinant) and acetylthiocholine (ATCh) were purchased from Sigma-Aldrich Co. LLC. (St Louis, MO, USA). DZO was purchased from Wako Pure Chemical Industries, Ltd. (Osaka, Japan). Ricinus communis agglutinin $\left(\mathrm{RCA}_{120}\right)$ was obtained from the Vector Laboratories (Burlingame, CA, USA). BTX/A/Hc was obtained from List Biological Labs, Inc. (Campbell, CA, USA). Ganglioside GT1b was purchased from IsoSep (Tullinge Sweden). Lyso-lactosyl ceramide was obtained from Avanti Lipids Polar, Inc. (Alabaster, AL, USA). Lactoside and GT1b derivatives were synthesized in a manner similar to that reported previously ${ }^{22}$. Au nanoparticles were supplied from Tanaka Precious Metals (Tokyo, Japan). Bovine serum albumin was purchased from Kokusan Chemical Co., Ltd. (Tokyo, Japan). The HBS-N buffer was $10 \mathrm{mM}$ of 4-(2-hydroxyethyl)-1piperazineethanesulfonic acid (HEPES) containing $150 \mathrm{mM}$ of $\mathrm{NaCl}$ (pH 7.5), filtered with a $0.2-\mu \mathrm{m}$ filter and degassed before use. All experiments were evaluated at room temperature.

Sarin, VX, and ricin are highly toxic, so these compounds were carefully handled using protective clothing within a fume hood and destroyed with sodium hypochlorite after experiments. BTX/ $\mathrm{A} / \mathrm{Hc}$ and $\mathrm{RCA}_{120}$ have no toxicity, but their handling was the same with the chemical and biological agents. The usage of sarin, VX, and ricin was approved by the Ministry of Economy, Trade and Industry of Japan ${ }^{24}$. Sarin and VX were made in-house at the National Research Institute of Police Science. Ricin was obtained from J-Oil Mills, Inc. (Tokyo, Japan).

\section{Design and fabrication of the air-sampling unit}

Our device contains three types of biosensors for the detection of different types of regents. These biosensors require that the sample reagent be dissolved in liquid (water) from the collected air because these biosensors use an enzyme and glycan chains that react or recognize in solution for detection. To dissolve the sample reagent in water efficiently, the sample reagents make contact with a mist of microdroplets. Sample reagents with the microdroplets in the air can be aspirated into the collection tube through the suction motor. Then, only the sample solution shall remain in the collection cylinder by gas/liquid separation ${ }^{25}$ (Figure 2a). To fulfill this concept, a sampling unit was designed. To generate the mist and spray it into the air, a mist generator consisting of $50 \mathrm{~mm} \times 53 \mathrm{~mm} \times 160 \mathrm{~mm}$ Polymethyl methacrylate (PMMA) material was designed and fabricated (Figure 2b). For the atomization of pure water, an ultrasonic vibration element (HM-2412; Honda Electronics Co., Ltd., Oscillating frequency: $2.4 \mathrm{MHz}$, input power source: DC $24 \mathrm{~V}$, atomization performance: $180 \mp 50 \mathrm{~mL} \mathrm{~h}^{-1}$, atomized particle size: approximately $3 \mu \mathrm{m}$ ) was placed at the bottom of the mist generator. The generated mist was released through the airflow of the DC fan motor (F310R-05LC; Copal Electronics Corp., power source: $5 \mathrm{~V}$ DC, Airflow: $0.09 \mathrm{~m}^{3} \mathrm{~min}^{-1}$ ), which was placed on the backside of the mist generator. Figure $2 \mathrm{c}$ shows the collection container. The collection container's size was $80 \mathrm{~mm}^{2} \times 82 \mathrm{~mm}$ high, and each collection cylinder in the container was $25 \mathrm{~mm}$ in diameter and $40 \mathrm{~mm}$ in height. The material used was PMMA. The collection cylinder has a hole along the center top wall where the outlet pipe $(6 \mathrm{~mm}$ in inner diameter, $8 \mathrm{~mm}$ in outer diameter, $20 \mathrm{~mm}$ in length) is connected. The sample reagents dissolved into microdroplets were sucked into the collection container after passing through the funnel that is connected to four collection cylinders. The cylindrical structure promotes a cyclone flow, which is well known to work as a small particle and gas separator due to the produced centrifugal force. The mist is concentrated, and it becomes larger droplets and gets attached along the inside wall of the cylinder. The concentrated droplets drip to the bottom of the cylinder, while the excess air gas heads outside through the center pipe in the cylinder. Thus, only the target solution remains in the cylinder bottom. The outlet channels from the cylinders converge to one outlet at the bottom side of the cartridge, as shown in Figure $2 \mathrm{c}$. This outlet is directly connected to the suction motor (three-phase brushless DC motor, CV-XG20; Hitachi, power source: 24 V DC, Tokyo, Japan). Our device works by battery power supply only, which is advantageous in the construction of a compact and portable device. Figures $2 \mathrm{~d}$ and e show the design and fabricated prototype of the air-sampling unit, respectively. The collection container is placed in the center and has four mist generators around it. The suction motor is placed and connected to the outlet in the bottom surface of the collection container. Figure $2 \mathrm{f}$ shows the motion of the air sampling with the generated mist at the same time.

\section{Construction of the automated detection device}

The collected sample solution in the cylinder must be transferred to all of the biosensor chips for the measurement of each agent. The reagents (i.e., AChE, enzyme substrates, buffers) also have to be kept in another space prior to performing the biosensing. We have designed the distribution unit parts that can fulfill these requirements. Moreover, the interlocking device system is built in coordination with the fabricated sampling unit to each biosensor chips, which can work automatically from sampling of the agents to reading and measurement. Transferring each solution into and from the cylinders was carried out by the combination of air pressure and valves. In detail, the mechanism of sample transferring is explained via the scheme in Supplementary Figure S1a as follows: the distribution cylinder has four silicon tubes (inner diameter of $1 \mathrm{~mm}$, outer diameter of $2 \mathrm{~mm}$ ) that are 


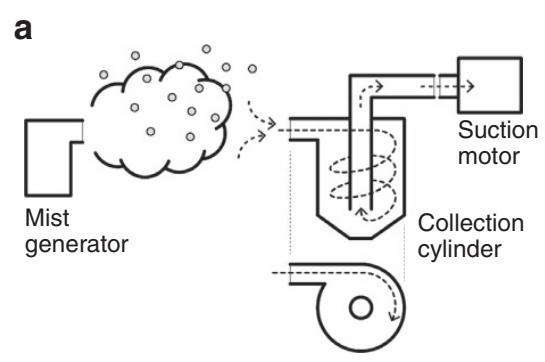

b
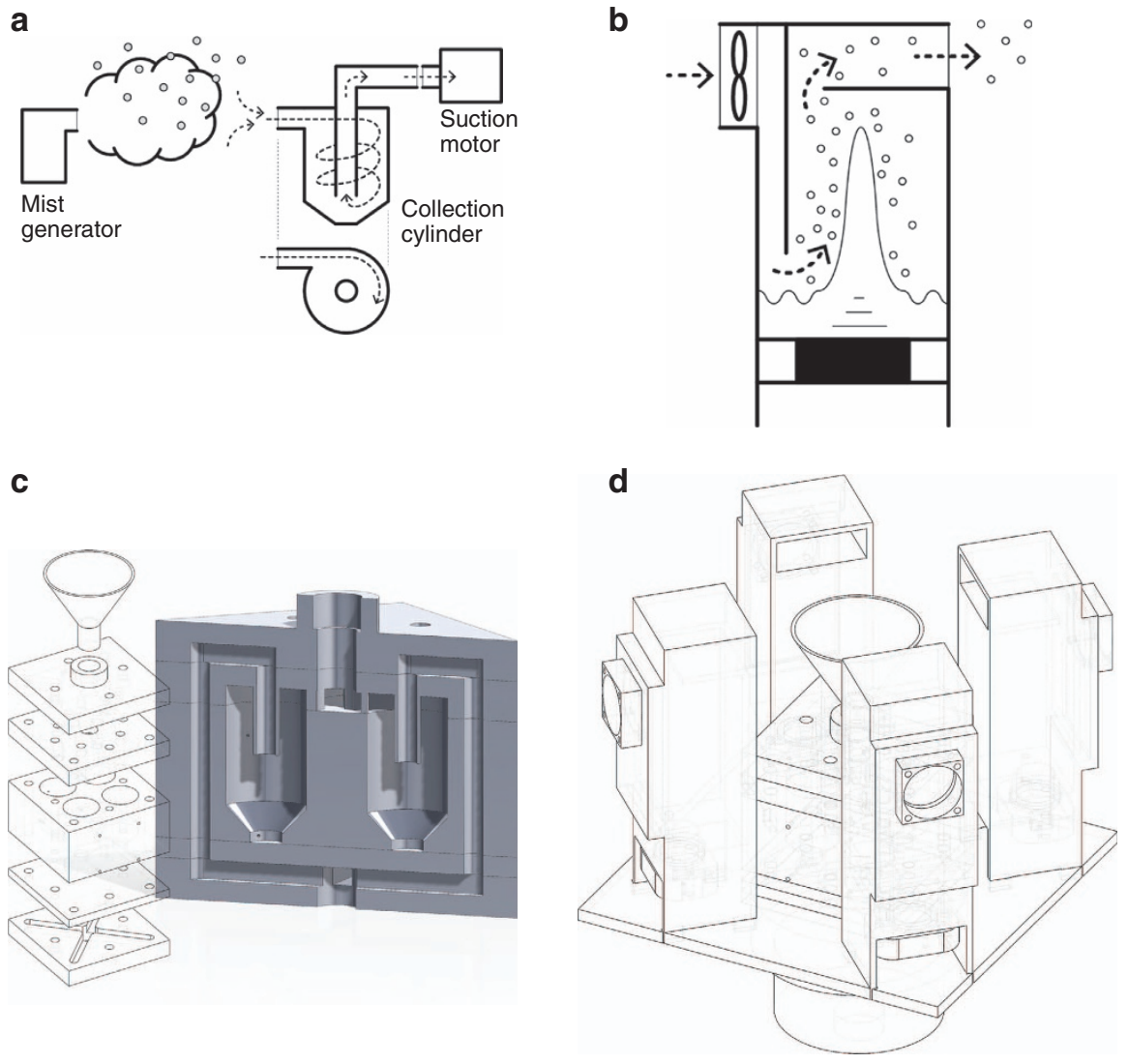

e

f
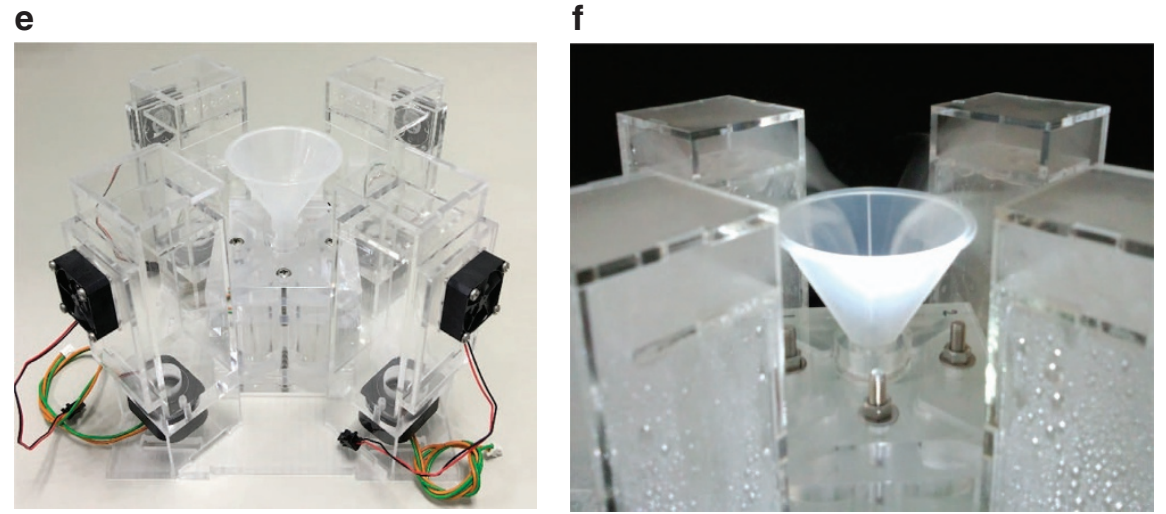

Figure 2 Developed air-sampling unit. (a) Scheme of air sampling. Samples in the air make contact with the generated water mist and get sucked into one of the collection cylinders. The air and mist are separated in the cylinder due to the cyclone motion, and the target solution will remain at the base of the cylinder due to gravity. (b) Mist generation scheme and design. The mist is generated by the ultrasonic transducer and spouted by the airflow from the DC fan motor. (c) Collection container composed of five-layered PMMA parts that are bolted together. The suction motor placed at the bottom of the collection container produces the intended airflow in the collection cylinders. (d) Design of the collection container and mist generator. (e) Prototype of the air-sampling unit; four mist generators and a collection container with a funnel. (f) View of the mist generation and air suction.

connected separately to the collection cylinder of the sampling unit, biosensor chip, and two small air pumps (Direct-current Air Pump CM-15-24; E.M.P Japan Ltd., Tokyo, Japan) for suction and extrusion. When the collected sample solution is transferred to the cylinder from the collection unit, valves 1 and 2 will be opened and valves 3 and 4 will be closed by the solenoids (DC solenoid $9 \mathrm{~mm}$ stroke push, 8.M12 40 62; Mecalectro) cramped to each silicon tube. In effect, the inner cylinder will have a negative pressure due to the suctioned air by the pump, and then the collected sample solution shall be pulled out into the distribution cylinder. To send the solution to the biosensor chip, valves 1 and 2 will be closed and valves 3 and 4 will be opened. The inner distribution cylinder will have a positive pressure by perfusion of the air, and then the sample solution will be delivered to the biosensor chip.

The entire connection network between the cylinders and other parts is shown in Supplementary Figure S1b. For the measurement of chemical agents, this unit has two compartments. One cylinder has dimensions of $8 \mathrm{~mm}$ in diameter and $20 \mathrm{~mm}$ in height and is used for stocking the ATCh substrate. Another cylinder with $8 \mathrm{~mm}$ in diameter and $30 \mathrm{~mm}$ in height has a screen-printed electrode at the bottom for electrochemical differential pulse voltammetry (DPV) measurement of enzyme-reacted solution. For measurement of a biological toxin agent, another two compartments were prepared. One cylinder is $14 \mathrm{~mm}$ in diameter and $35 \mathrm{~mm}$ in height 
and is for storing and sending the running buffer to take the baseline to the LSPR biosensor chip. Another cylinder that is $14 \mathrm{~mm}$ in diameter and $35 \mathrm{~mm}$ in height is used for transferring and sending the collected solution to the LSPR biosensor chip. For measurement of the genes of biological agents, another cylinder that is $14 \mathrm{~mm}$ in diameter and $35 \mathrm{~mm}$ in height was designed for storing and sending the PCR reagent.

The sampling time for chemical agent detection was set to $1 \mathrm{~min}$. AChE, which was stocked in the collection cylinder in advance, was reacted with the collected sample and the sprayed mist during air sampling in the collection cylinder. The stocked ATCh substrate in the distribution unit gets sucked into the collection cylinder by opening the valve for $50 \mathrm{~s}$ from the start of air sampling. For the remaining $10 \mathrm{~s}$ of air-sampling time, the substrate solution reacts with $\mathrm{AChE}$ by mixing in the cyclone motion inside the collection cylinder. AChE reacts with the ATCh substrate for $110 \mathrm{~s}$ continuously, with a total reaction time of $120 \mathrm{~s}$. If the collected sample contains the chemical agent, AChE reaction is inhibited. The reacted solution is transferred to the sensor electrode and measured by DPV. In the case of biological toxin detection by the LSPR sensor, a blank buffer flows initially to the LSPR sensor chip to take the baseline for $200 \mathrm{~s}$. Then, after air sampling for $1 \mathrm{~min}$, the collected sample solution is transferred into the cylinder of the distribution unit, which is stocked with the blank buffer to dilute the collected sample solution by bubble mixing. After a lapse of $200 \mathrm{~s}$, the collected sample solution runs to the LSPR sensor by switching from the blank buffer. For biological pathogen gene detection, the collected sample solution is transferred into the cylinder of the distribution unit and then mixed with the PCR solution, which is stocked in advance. This mixing is performed via the flowing air that is generated by repeated opening and closing of the valves. The PCR solution is sent to the sample divider, split as necessary into different volume amounts, and finally injected into the PCR chip for biological gene detection.

The parts described above were integrated with the airsampling unit as shown in Supplementary Figure S2. In addition, a control system to operate the integrated device was also constructed (Supplementary Figure S3). The electrical circuit for supplying the power source and switching on/off the device parts (i.e., the solenoid, DC fan motor) was fabricated by outsourcing (ESPEC Test System Corp., Kobe, Japan and MICRONIX Inc., Kyoto, Japan). The software program was coded using LabVIEW (National Instruments Japan Corporation), and the system was controlled through the input-output signal of a USB DAQ (USB-6216; National Instruments Japan Corporation). This device was also designed to work with a 24-V battery, which is beneficial for on-site detection utility.

Electrochemical DPV measurement for chemical wafer agents An SPCE (S6) and a USB-powered potentiostat were purchased from Biodevice Technology, Ltd. (Ishikawa, Japan). Instrumental parameters of the DPV were as follows: the potential range from 0 to $1 \mathrm{~V}$ was scanned with a step potential of $10 \mathrm{mV}$, the pulse amplitude was $250 \mathrm{mV}$, the pulse period was $500 \mathrm{~ms}$, the pulse width was $50 \mathrm{~ms}$, the sampling width was $1 \mathrm{~ms}$, and the scan rate was $20 \mathrm{mV} \mathrm{s}^{-1}$

\section{LSPR measurement using our device}

The conditions used for the LSPR measurement settings have been described in the previous report ${ }^{22}$. Glycochips for the LSPR detections were made in the same manner as in our previous report. The glycochip was set into the flow cell and connected to silicone and polytetrafluoroethylene tubes. About $10 \mathrm{mM}$ of HEPES buffer ( $\mathrm{pH}$ 7.5) containing $150 \mathrm{mM}$ of $\mathrm{NaCl}$ was used as a running buffer. A volume of $3 \mathrm{~mL}$ of HEPES buffer was added in the chamber of the baseline side, while $1 \mathrm{~mL}$ was added in the chamber of the collected solution. A $5 \mathrm{~mL}$ volume of biological toxin or simulant solution was set in the atomizer container to be sprayed. The sprayed amount of biological toxin was measured by comparing the volume weight change of the atomizer container after the experiment. This was compared to the volume weight of the residual solution amount at the collected chamber corresponding to the LSPR response to generate the standard curves from which the recovery yield was calculated. The absorbance spectrum or signals for LSPR measurement were obtained using the free software OOIBase 32 . The absorbance change was obtained as a subtraction at 720 and $550 \mathrm{~nm}$.

\section{Pathogen detection for PCR}

Forward and reverse primers and a TaqMan probe were purchased from Thermofisher, and the sequences were 5'-TCGGATC GCAGTCTGCAA, 5'-GCATGCTGATCCGCGATTA, and 5'-FAM-CGACT GCGTGAAGCTGGAATCGC-TAMRA, respectively. SpeedSTAR HS DNA polymerase was purchased from Takara Bio Inc. (Tokyo, Japan). A standard solution of Bacillus subtilis (BS) spore was obtained from Eiken Chemcial Co., Ltd. (Tokyo, Japan). A segmentflow PCR chip with a serpentine microchannel was fabricated from cycloolefin polymer by injection molding and sealing with a transparent thin film (3 M 9795). A coating solution was injected into the microchannel and removed by an aspirator. The coating solution consisted of a PCR mixture for BS except for template DNA; $3 \mu \mathrm{M}$ of each primer, $0.2 \mu \mathrm{M}$ of TaqMan Probe, $0.3 \cup \mu \mathrm{L}^{-1}$ of SpeedStar HS DNA polymerase. The segment-flow chip, which was stored in the dark at room temperature, was fixed on two pieces of aluminum blocks with cartridge heaters included. These blocks were heated at $98^{\circ} \mathrm{C}$ for denaturation and $58^{\circ} \mathrm{C}$ for annealing and extension. A volume of $0.3 \mathrm{~mL}$ of PCR reagents, which was the same as the contents in the coating solution, was preserved in a reservoir of a developed aerosol sampler. The BS spore solution was sprayed by an atomizer around the inlet of the aerosol sampler and was collected by surrounding mist flowing into the reservoir. The collected aerosol solution was mixed with the PCR reagents during vigorous air blowing into the reservoir. The mixed PCR solution flowed to a sample injector through a connected tube. A volume of $11 \mu \mathrm{L}$ of the PCR solution was distributed into the segment-flow PCR chip and thermally treated while flowing through the serpentine microchannel. The PCR solution repeatedly and successively made contact with each temperature zone from two different heat blocks. The fluorescence intensity was then measured at the end of the microchannel by a fiber fluorescence detector (Nihon sheet glass, Japan).

\section{RESULTS AND DISCUSSION}

\section{Construction of the aerosol-sampling unit}

The device contains three types of biosensors for the detection of different types of reagents. These biosensors require the target reagent to be dissolved in water because of the presence of the enzymes and carbohydrate chains that react or bind in the solution for detection. Biocapture650 is well known as a representative example of aerosol-sampling equipment. However, it is difficult to operate our biosensors with the commercially available sampler since the collection cartridge contains detergent in advance, which limits the utility of the three biosensing technologies. Wanting a design with compact portability for onsite detection and with air-suction capability for the collection of a large volume of air and dissolving of the sample agents into a solution for increased sensitivity, a sampling unit was designed and developed. Figures $2 \mathrm{~d}$ and e show the complete air-sampling unit prototype.

The aerosol was formed by having a mist of sterile microdroplets, formed by the mist generator (Figure 2b), make contact with the ambient air. The high surface-area-to-volume ratio of 
droplets promotes the easy collection and efficient dissolution of the particulates in the air. The sample preparation assembly consists of four mist generators and one central collection funnel (Figure 2f). After passing through the central channel, the mixture is drawn into four collection tubes where the target solution remains by gas/liquid separation due to the cyclone effect in a cylindrical hollow geometry ${ }^{25}$ (Figure 2a). The cyclone effect promotes the formation of larger droplets inside the collection tubes and applies a centrifugal force to make the droplets stick to the inside wall. The droplets containing the target sample then drip to the bottom of the collection cylinder and are subsequently delivered to the biosensors. The excess air flows outside through the center pipe in the collection cylinder (Figure 2c). Compared to Biocapture650, which has a collection rate of $2001 \mathrm{~min}^{-1}$, the measured flow rate in the developed air-sampling unit was $338 \mathrm{I} \mathrm{min}^{-1}$, which is 1.7 times higher.

In addition to the comparable performance of the developed device, the components run with a $24-\mathrm{V}$ battery power supply only. Having electrical parts that work with a direct-current power source only, the construction of a compact and portable device was easily realized.

\section{Detection of chemical warfare agents}

AChE inhibition is the most well-known mode of action of nerve gas agents. The inhibition mechanism is well studied; organophosphates bind to the catalytic site of AChE irreversibly ${ }^{4}$. Some organophosphate pesticides, such as Diazinon oxon (DZO, diethyl 2-isopropyl-6-methyl-4-pyrimidinyl phosphate), can also inhibit AChE activity via a similar mechanism. As one of the analytical methods, electrochemical measurements for AChE inhibition have been widely reported ${ }^{26,27}$. Our research group has also reported a colorimetric sensor for the detection of DZO based on the inhibition of $\mathrm{AChE}^{28}$. Additionally, we have developed a portable electrochemical detection method using a USB-powered potentiostat and a disposable SPCE ${ }^{19-21}$. With these, we integrated our electrochemical biosensing device for chemical agent detection due to its portability and mobility. For initial optimization of electrochemical conditions, DZO was carried out as the simulant to reduce the risk of poisoning because the toxicity of DZO is approximately 100 times less than that of sarin ${ }^{29}$. ATCh was used as a substrate for measurement of AChE activity ${ }^{30,31}$. Thiocoline (TCh) was enzymatically produced and measured by electrochemical oxidation signals derived from the DPV technique (Figure 3a).

A volume of $20 \mu \mathrm{L}$ of $4 \mathrm{U} \mathrm{mL}^{-1}$ AChE (in PBST, pH 7.4) and $50 \mu \mathrm{L}$ of different concentrations of DZO (diluted with ultra-pure water) were mixed in a $1.5-\mathrm{mL}$ tube for $50 \mathrm{~s}$ to inhibit the AChE activity. After inhibition of the AChE activity, $20 \mu \mathrm{L}$ of $100 \mathrm{mM}$ ATCh (in ultra-pure water) was added to the mixture to generate thiocoline for $2 \mathrm{~min}$. A volume of $20 \mu \mathrm{L}$ from the reaction mixture was immediately applied to SPCE, and the AChE activity was measured using the DPV mode of the USB-powered potentiostat (Figure $3 \mathrm{~b}$ ). Figure $3 c$ shows the DPV voltammograms of the mixture with various concentrations of DZO. The DPV response from thiocoline oxidation could be observed at approximately $0.5 \mathrm{~V}$ of the peak potential without DZO inhibition. Oxidation current values decreased greatly corresponding to the increment of the DZO concentrations to $2 \times 10^{-5} \mathrm{~g} \mathrm{~mL}^{-1}$. At $5 \times 10^{-5} \mathrm{~g} \mathrm{~mL}^{-1}$ of DZO, the DPV response could hardly be observed. Thus, the peak height of the oxidation current was determined. A straight line between the two ends of the signal peak was drawn as the baseline, and the peak height was calculated (Figure 3d). To indicate the degree of AChE activity inhibition, the reduction of the peak current height with AChE inhibition by chemical agents was compared with the peak current height without AChE inhibition and represented as the relative inhibition (\%) rate. The DZO concentration dependence of the AChE inhibition was estimated, and the standard curve is presented in Figure 3e. A concentration of $10 \mathrm{ng} \mathrm{mL}^{-1}$
DZO indicated $5.8 \pm 5.6 \%$ of slight relative inhibition. With the increment of DZO concentration, a relative inhibition of $97.9 \%$ was observed for $50 \mathrm{\mu g} \mathrm{mL}^{-1}$.

The inhibition of AChE activities with VX and sarin was also measured using the same method. In the case of AChE inhibition by sarin, the relative inhibition reached $94.7 \%$ for a high concentration of $100 \mathrm{ng} \mathrm{mL}^{-1}$. A concentration of $0.3 \mathrm{ng} \mathrm{mL}^{-1}$ sarin was reliably detected with $8.5 \%$ relative inhibition, which is lower than the minimum DZO concentration detected. This is highly associated with the toxicity of sarin, which is 100 times higher than that of the organophosphate pesticide $\mathrm{DZO}^{29}$.

In the case of $\mathrm{VX}$, the relative inhibition was $96.1 \%$ at $30 \mathrm{ng} \mathrm{mL}^{-1}$ and $12.9 \%$ at $0.3 \mathrm{ng} \mathrm{mL}^{-1}$. The standard curve of VX inhibition almost overlapped that of sarin inhibition. The molecular weights of VX and sarin are 267.4 and 140.1, respectively. VX had the same inhibition at approximately half the number of molecules compared with sarin. This result simply means that VX inhibition was more sensitively detectable. In addition, VX is 240 times more toxic than DZO and 2.4 times more toxic than sarin. From these results, we confirmed the applicability of our electrochemical device to obtain the response depending on the inhibition activity and concentrations.

After optimizing the electrochemical parameters, we evaluated our electrochemical device together with the air-sampling unit based on the AChE inhibition of the collected chemical warfare agents in the capture cylinder. The work flow was as follows: an aqueous solution of a chemical warfare agent was sprayed in the air using an atomizer, ranging from $1 \mathrm{ng}$ to $1 \mu \mathrm{g}$ for $1 \mathrm{~min}$. For another $1 \mathrm{~min}$, the mist from the generator interacts with the atmosphere and then enters the collection funnel. The AChE was stocked in the collection cylinder in advance. The solution is mixed in the cylinder by the cyclone motion of the air during the airsampling step. Afterwards, the substrate solution was supplied into the collection cylinder from the distribution container after a lapse of $50 \mathrm{~s}$. Enzyme reaction was maintained for $2 \mathrm{~min}$, and then the mixture was sent to the SPCE for electrochemical measurement. The atomizer for spraying the chemical warfare agents and the device were placed in a custom-made cage covered with a vinyl sheet, and all experiments were carried out in this controlled environment. Details on controlling the distribution of solutions after the air-sampling step are described in the Materials and methods section and illustrated in Supplementary Figures S1-S3. Note that all steps are done automatically.

The results (Figure $3 \mathrm{f}$ ) show that sarin could be detected with great certainty at a minimum amount of $30.0 \mathrm{ng}$ with relative inhibition of $23.5 \pm 2.8 \%$ (C.V. $11.7 \%$ ). At more than $1.17 \pm 0.06 \mu \mathrm{g}$, the relative inhibition was $93.7 \pm 2.44 \%$ (C.V. $2.6 \%$ ). In the case of VX, the relative inhibition was $47.2 \pm 5.3 \%$ (C.V. $11.2 \%$ ) for a low amount of $86.6 \pm 0.24 \mathrm{ng}$. A lower amount of $36.2 \mathrm{ng} V X$ could be detected; however, measurement was performed only once. The recovery rate was defined as the ratio of the amount of sprayed chemical warfare agent to that of the amount collected in the cylinder. The amount of the collected chemical warfare agent was calculated from the standard curve of relative inhibition, which was estimated from the sensor capability in advance (Figure 3e). Calculations show that the sprayed $1.2 \pm 0.05 \mu \mathrm{g}$ sarin and $1.2 \mu \mathrm{g}$ VX had recovery rates of $13.1 \pm 6.9$ and $3.24 \%$, respectively. This difference is associated with the solubility of the reagents to water; sarin has a $100 \%$ solubility at $25^{\circ} \mathrm{C}$, while VX has only $3 \%$ solubility ${ }^{32}$. Though the recovery rate is poor, the sensitivity of the electrochemical detection is sufficient to compensate this innate problem. The $\mathrm{LD}_{50}$ (50\% of the lethal dose) of sarin and VX is 150 and $40 \mathrm{mg} \mathrm{m}^{-3}$ (respiratory minute volume for humans), respectively ${ }^{7}$. When $30 \mathrm{ng} \mathrm{VX}$ was sprayed in the atmosphere, the density in the atmosphere was expected to be $90 \mathrm{ng} \mathrm{m}^{-3}$, which is completely below the $\mathrm{LD}_{50}$ but detectable by the device. Our developed system could achieve automatic detection rapidly, proceeding from the air-sampling step to electrochemical sensing 

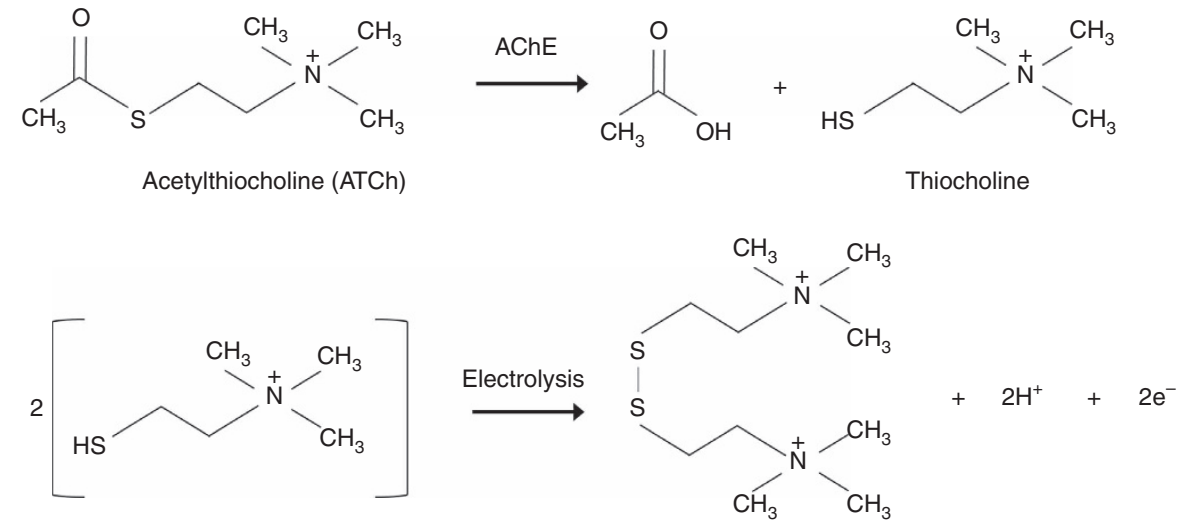

Dithiobischoline

\section{b}
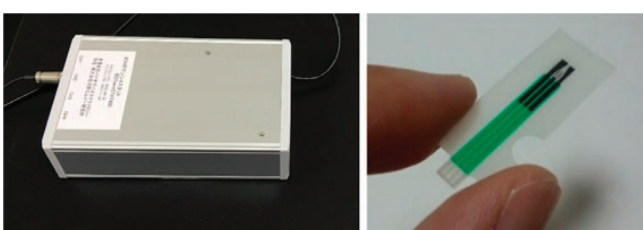

C

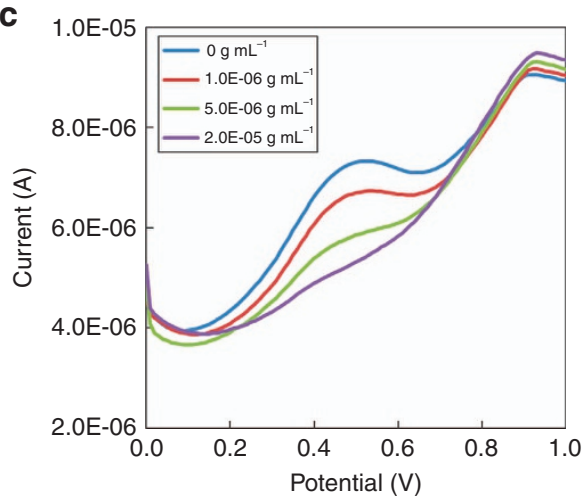

e

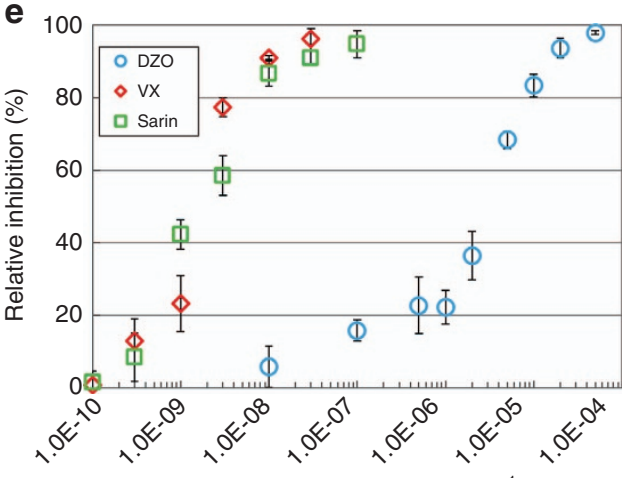

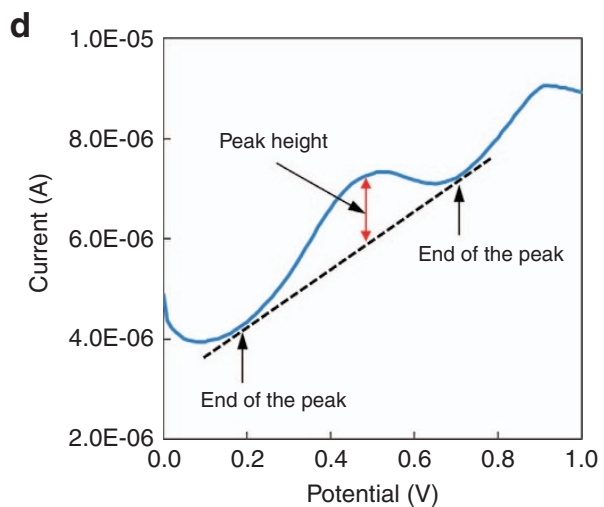

f

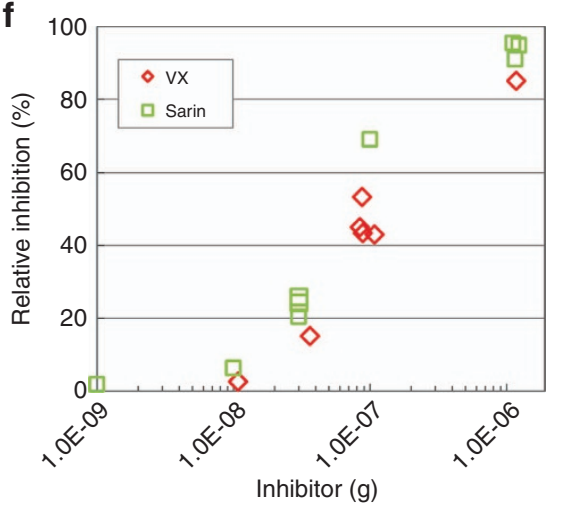

Figure 3 Detection of chemical warfare agents with the electrochemical method. (a) Scheme of the electrochemical measurement of the inhibitory activity of AChE. (b) USB-powered potentiostat and screen-printed carbon electrode. (c) DPV voltammograms of the mixture with 0-2.0 E-5 g mL ${ }^{-1}$ of DZO. (d) The peak height of the oxidation current determines the baseline of correction. (e) The relation between AChE inhibition and chemical warfare agent concentrations as a standard curve obtained by plotting the peak current of DPV measurement. (f) Estimation of autonomous measurement by our system through the collection of chemical warfare agents in air to electrochemical measurement of AChE inhibition.

within $5 \mathrm{~min}$. The collected solutions in the cylinder were also analyzed by a large-volume injection of GC-MS, which confirmed the presence of the chemical agents (data not shown). In addition, to see the specificity of the device and that contaminants in the field do not interfere with the measurements, the atmosphere in the university parking lot was tested and measured with the sensor. No influence was seen in the obtained measurements (Supplementary Information). 
The limit of detection (LOD) of our developed system was compared with other mobile methods for measuring nerve agents based on the published results ${ }^{7}$. A Draeger gas detection tube indicated a $0.1 \mathrm{mg} \mathrm{m}^{-3} \mathrm{LOD}$, an ion mobility spectrometer has a $0.2 \mathrm{mg} \mathrm{m}^{-3} \mathrm{LOD}$, and a flame photometric detector also has a $0.1 \mathrm{mg} \mathrm{m}^{-3}$ LOD. The sensitivity of our developed system achieved detection at nanogram order and was far lower compared to the existing mobile devices.

\section{Detection of toxin agents}

For detection of biological toxins, commercially available detection kits and systems have been developed, and in most cases, they usually require ca. 15-30 min for detection aside from the sample preparation when suspected materials are examined. These methods require sample preparation manually at the scene of the incident by the first responders while wearing heavy biohazard suits, which is difficult to handle, resulting in often tedious and time-consuming operation. Moreover, the kits and systems available are suited for a powder or a liquid sample only and not for an aerosol. Among many envisaged terror scenes, inhalation can cause the most considerable damage to the population. Therefore, suspected biological clouds in the air, which may contain toxins or threat agents, should be assessed conveniently and rapidly with minimum or no manual sample preparation.

In the present paper, the suspected cloud or aerosol containing the threat biological warfare agents is targeted, and a fully automated biohazard detection system composed of sample collection, detection, and signal display has been developed, in which no sample preparation, such as swab collection and processing by the first responder, is required.

Botulinum toxins (BTX) from Clostridium botulinum are extremely poisonous proteins occurring in nature. The BTXs are ranked as 'category A' by the Centers for Disease Control and Prevention (USA) and have gained increased attention in risk management and counter-measures against bioterrorism. Among seven different serotypes ( $A$ to $G$ ) of the botulinum toxins, type $A$ has the highest toxicity: $L_{50}$ is in the range of $0.7-0.9 \mu \mathrm{g}$ per human in inhalation. In our study, we selected the type $A$ toxin as the main target, which has two components, a light chain (MW $50 \mathrm{kDa}$ ) and a heavy chain (MW $100 \mathrm{kDa}$ ). They are linked together by a disulfide $(\mathrm{S}-\mathrm{S})$ bonding. The light chain $(\mathrm{LC})$ has a protease activity a

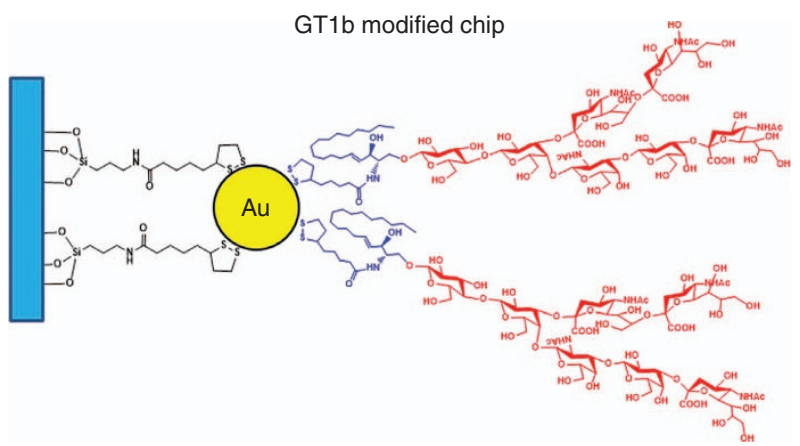

C

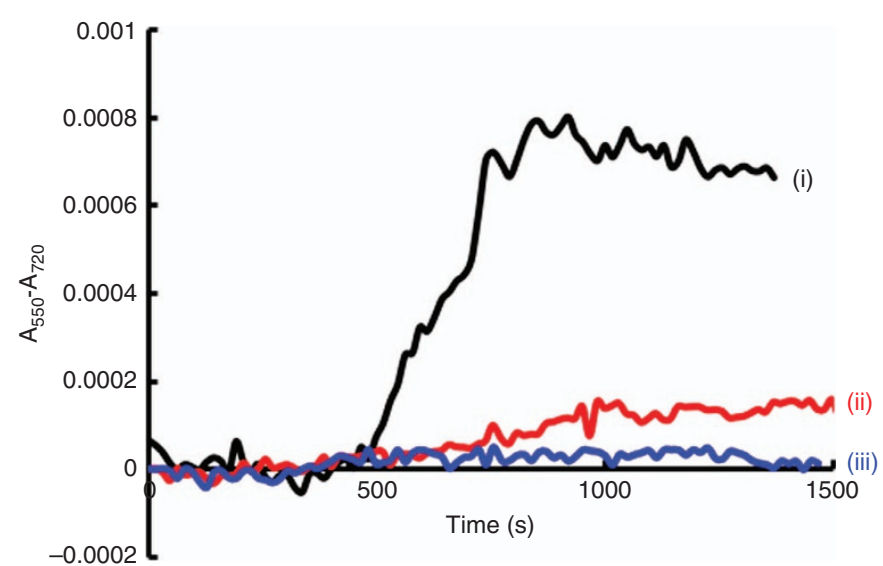

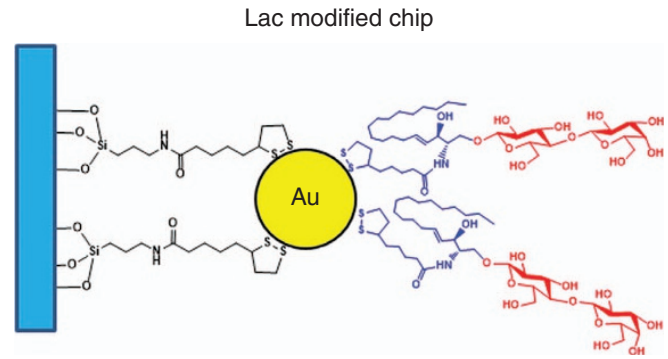

d

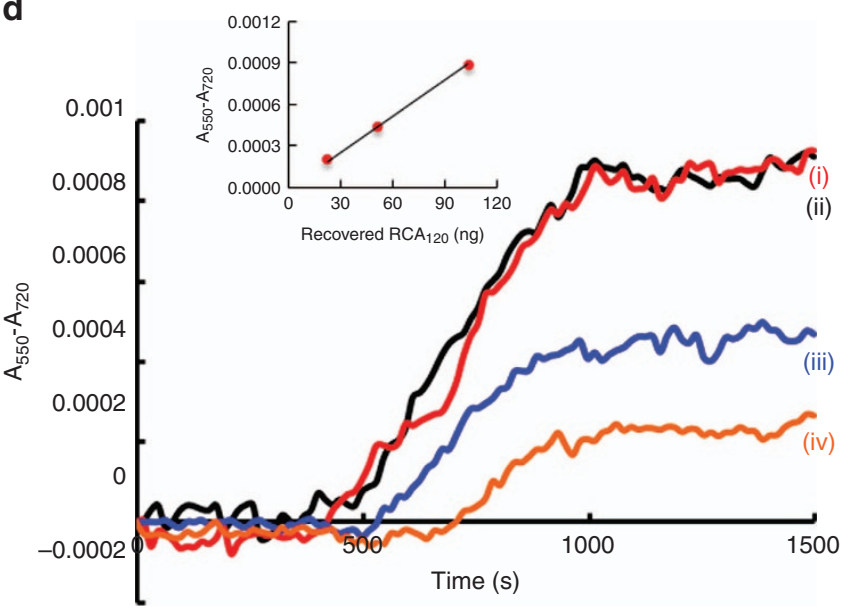

Figure 4 Structures of LSPR chips for detection of (a) botulinum toxin (BTX) and (b) ricin. For BTX detection chips, 40-nm Au nanoparticles were immobilized, while for ricin detection, $20 \mathrm{~nm}$ ones were used. These chips and the synthetic glycolipids as the toxin ligands were prepared based on our previous paper ${ }^{22}$. (c) LSPR detection of BTX/A/Hc with GT1b-modified LSPR chips after BTX/A/Hc collection by our device. (i) ca. $1 / 15$ of the equivalent of $B \operatorname{BX}_{50}(2 \mu \mathrm{g})$, (ii) ca. $1 / 45$ of $L D_{50}$ equivalent $(0.83 \mu \mathrm{g})$. (iii) Bovine serum albumin at approximately $1 / 15$ of the equivalent BTX $\operatorname{LD}_{50}(2 \mu \mathrm{g})$ as the negative control. Here, $\mathrm{LD}_{50}$ in inhalation was calculated based on a 70-kg human that inhales the air contaminated with BTX/A for $15 \mathrm{~min}$. Detection chip: GT1b-functionalized chip, Target toxin: BTX/A/Hc, Flow rate: $138 \mathrm{~mL}$ min ${ }^{-1}$, Running buffer: $10 \mathrm{mM}$ HEPES ( $\mathrm{pH} 7.5)+150 \mathrm{mM} \mathrm{NaCl}$. Each recovery rate of BTX/A/Hc collected in sample bottles in the system was calculated from the amounts of $151.4 \mathrm{~mL}$ of sprayed solution, and each toxin concentration was determined from the standard calibration curve. Recovery of $76 \%$ for (i) and $53 \%$ for (ii). (d) LSPR sensorgram of (i) ricin at approximately $1 / 45000$ of $L_{50}(0.23 \mu \mathrm{g}$ ), and RCA 120 at (ii) ca. $1 / 90000$ of $\operatorname{LD}_{50}(0.12 \mu \mathrm{g})$, (iii) ca. $1 / 220000$ of $\operatorname{LD}_{50}(0.06 \mu \mathrm{g})$, and (iv) ca. $1 / 440000$ of $\operatorname{LD}_{50}(0.03 \mu \mathrm{g})$. The inlet picture in (d) is the relation dose-response plots between the LSPR response and recovered amounts of RCA $A_{120}$. Here, $L D_{50}$ of ricin from inhalation was calculated based on a 70-kg human that inhales the air contaminated with ricin for 15 min. Detection chip: Lac chip, Target toxins: ricin, Flow rate: $138 \mathrm{~mL} \mathrm{~min}^{-1}$, Running buffer: $10 \mathrm{mM}$ HEPES (pH 7.5)+150 mM NaCl. 
to cleave the SNAP-25 and inhibit acetyl choline release. The heavy chain $(\mathrm{Hc})$ has a carbohydrate binding domain and specifically attaches the glycolipids called GT1b gangliosides on human nerve cells.

Here, we applied the natural recognition and binding process of this toxin for selective and rapid detection. As a transducer detection device, the LSPR biosensor was used. A GT1b ganglioside mimic was designed and synthesized from a natural GT1b ganglioside. The synthetic GT1b derivative carries lipoic acid for attachment on Au surfaces, and an LSPR chip modified with GT1b was assembled with our established methodology ${ }^{22}$. As illustrated in Figure 4a, the derived GT1b LSPR chip utilized Au nanoparticles of $40 \mathrm{~nm}$ in size. In the present study, a heavy chain binding domain $(\mathrm{BTX} / \mathrm{A} / \mathrm{Hc})$ was used instead of the neurotoxins (BTX/A) composed of $\mathrm{Hc}$ and Lc with potent toxicity.

To assess the LSPR biosensor unit, we examined the potential utility of the biosensor using different concentrations of $R_{C A} A_{120}$, ricin $\left(R A_{60}\right)$ and $B T X / A / H c$ in solution. Supplementary Figure S5 indicates the linear relationship between the LSPR responses and these reagent concentrations. The calibration curve shows the linearity ranging from 0 to $200 \mathrm{ng} \mathrm{mL}^{-1}$, indicating that the present LSPR unit is reliable. Furthermore, for the ricin concentration of $1 / 3000$ with the measured LSPR response of $0.000805 \pm 0.000196$, the C.V. value was $25 \%(n=3)$, which demonstrates the reliability of the measurements.

Figure $4 c$ indicates that LSPR detection of $B T X / A / H c$ with the GT1b-modified chip after BTX/A/Hc from aerosol generated using an atomizer was collected by the integrated portable biosensor. To facilitate the detection of toxins, the flow system was controlled in the following manner. The running buffer was first pumped for $200 \mathrm{~s}$ into the flow channel to establish the baseline, the collected sample was then pumped for $600 \mathrm{~s}$, and the pump was then switched back to the running buffer (details in Supplementary Table S1 and Supplementary Figure S6). Consequently, clear absorbance changes were obtained in the LSPR sensorgrams. Here, $\mathrm{LD}_{50}$ in inhalation is ca. $700 \mathrm{ng}$ when a $70-\mathrm{kg}$ human inhales air contaminated with $B T X / A^{33}$. Because humans inhale ca. $8 \mathrm{I}$ of air in $1 \mathrm{~min}, L_{50}$ is estimated to be ca. $0.7 \mu \mathrm{g} / 8 \mathrm{I}$ for $1 \mathrm{~min}^{34,35}$. This condition limit of inhalation for $1 \mathrm{~min}$ was set, which corresponds to the air contaminated with the toxin of ca. $87.5 \mathrm{\mu g} \mathrm{m}^{-3}$. Because the collection unit aspirates $338 \mathrm{I}$ of air for $1 \mathrm{~min}$, the system inhales ca. $30 \mu \mathrm{g}$ of $\mathrm{BTX} / \mathrm{A} / \mathrm{Hc}$ for $1 \mathrm{~min}$. In contrast, ca. $2.0 \mu \mathrm{g}$ of BTX/A/Hc was sprayed from the atomizer in the form of mist contaminated with the toxin, which corresponds to $\mathrm{LD}_{50}$ in inhalation. As a result, the integrated portable biosensor was able to collect and capture ca. $1.6 \mu \mathrm{g}$ of $\mathrm{BTX} / \mathrm{A} / \mathrm{Hc}$ from the contaminated aerosol. The collected air containing BTX/A/Hc was mixed with pure water in the collection cylinder to recover the target toxin and then flowed to the GT1b-modified chip on the LSPR system automatically for toxin detection. In this case, recovery of the toxin was determined to be $76 \%$ (calculation details are shown in the Materials and methods section) based on the LSPR calibration curve (Supplementary Figure S5b), meaning that the system effectively collected the toxin. In Figure $4 c(i)$, the LSPR response reached saturation after ca. $800 \mathrm{~s}$ (the sample was injected at $0 \mathrm{~s}$ ), showing that the concentration of the equivalent BTX/A LD 50 inhalation could be detected. In addition, the present system could detect a lower concentration of $1 / 45$ of the $\mathrm{LD}_{50}$ equivalent of the BTX/A (Figure 4c(ii)), making the present system highly sensitive.

Next, we examined ricin detection. Ricin is classified as a category- $B$ biological agent and is less hazardous than those in category A. Ricin has two subunits: the A-subunit has ribonucleic activity, and the B-subunit has two carbohydrate binding domains. The B-subunit binds to the $\beta$-D-galactopyranosyl ( $\beta$-D-Gal) residue that we utilized for the detection of this toxin. The LSPR chip for the ricin detection is shown in Figure $4 \mathrm{~b}$, and the lactoside having the $\beta$-D-Gal residue-modified chip was prepared as reported previously $^{22}$. For detection of ricin, $20 \mathrm{~nm} \mathrm{Au} \mathrm{nanoparticles} \mathrm{were}$ used (Figure 4b). When the $1 / 45000$ equivalent (ca. $0.23 \mu \mathrm{g} \mathrm{ml}^{-1}$ ) of ricin $\mathrm{LD}_{50}$ of inhalation was sprayed, the integrated system collected the toxin into the water, and then ricin was clearly detected (Figure $4 d(i))$. The result shows that the system can detect the toxin at a much lower concentration of $L D_{50}$ of inhalation; therefore, the present system will be useful as an onsite tool for simultaneous detection of chemical and biological agents even if the toxin has been diluted in air. Thus, to evaluate the limitation of the present system as primary study for toxins, the same experiment was carried out for concentration dependency using a ricin simulant, $R C A_{120}$. A concentration of $0.66 \mu \mathrm{g} \mathrm{ml}^{-1}$ of $\mathrm{RCA}_{120}$, which corresponds to $1 / 90000$ of the $\mathrm{LD}_{50}$ of ricin, was sprayed and collected from the aerosol for $1 \mathrm{~min}$ using our apparatus. The recovery yield was calculated to be $83 \%$. This numeric value was much higher than that of the other detected signals. The high recovery is associated with the biological toxin's solubility in water and its molecular weights being higher than those of the chemical warfare agents. Lower concentrations of $\mathrm{RCA}_{120}$ (ca. 1/220 000 and ca. 1/440 000 of the $\mathrm{LD}_{50}$ ) were also evaluated, and the obtained recovery yields were 80 and $75 \%$, respectively. As shown in Figure 4d, the plots of the recovery weights of $R C A_{120}$ and LSPR responses showed almost linear dose-response relationships; this indicated that our developed device exhibited good performance. In the LSPR sensorgram, 1/45 000 concentration of ricin of $\mathrm{LD}_{50}$ in inhalation showed a response similar to that of $1 / 90000$ concentration of $\mathrm{RCA}_{120}$. This result is attributed to the fact that the molecular weight of ricin is half of that of $\mathrm{RCA}_{120}$, and the number of sugarbinding sites of ricin is also half of that of $\mathrm{RCA}_{120}$.

\section{Detection of pathogen agents}

We also evaluated our device for the detection of pathogen agents from the air sample using the installed rapid on-chip PCR device. BS spores were used as a simulant. The performance of our rapid on-chip PCR technology was previously reported ${ }^{23}$. A small volume of PCR solution was injected in the microchannel and formed the segment-flow through the perfused air and vapor pressures. When $2.5 \times 10^{6}$ cfu of the BS spore simulant solution was collected by the generated mist and became concentrated in the reservoir, the estimated amount of the collected BS spores was $1.6 \times 10^{5} \mathrm{cfu}$. The calculated concentration was approximately equivalent to $800 \mathrm{cfu} \mathrm{uL}^{-1}$. Figure 5 shows the results of the amplified fluorescence intensity after the segment-flow PCRs with the various concentrations of BS spores. The flow rate of the PCR solution was adjusted to detect low concentrations of positive control. The flow rate was fixed at $24 \mu \mathrm{L} \mathrm{min}^{-1}$, and the total reaction time was 12.5 min for 40 cycles of the segment-flow PCR. Orange-colored bars indicate the fluorescence intensities after the

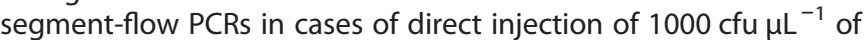
BS spore solution and no template control (NTC) solution without the aerosol sampler: positive and negative controls, respectively.

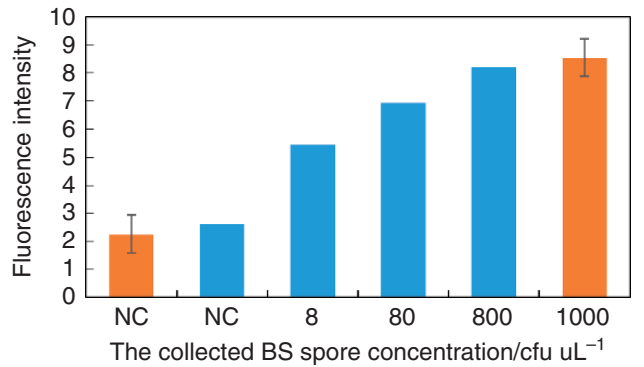

Figure 5 Estimation of our device to detect pathogen simulant from the air sampling to on-chip PCR detection. 

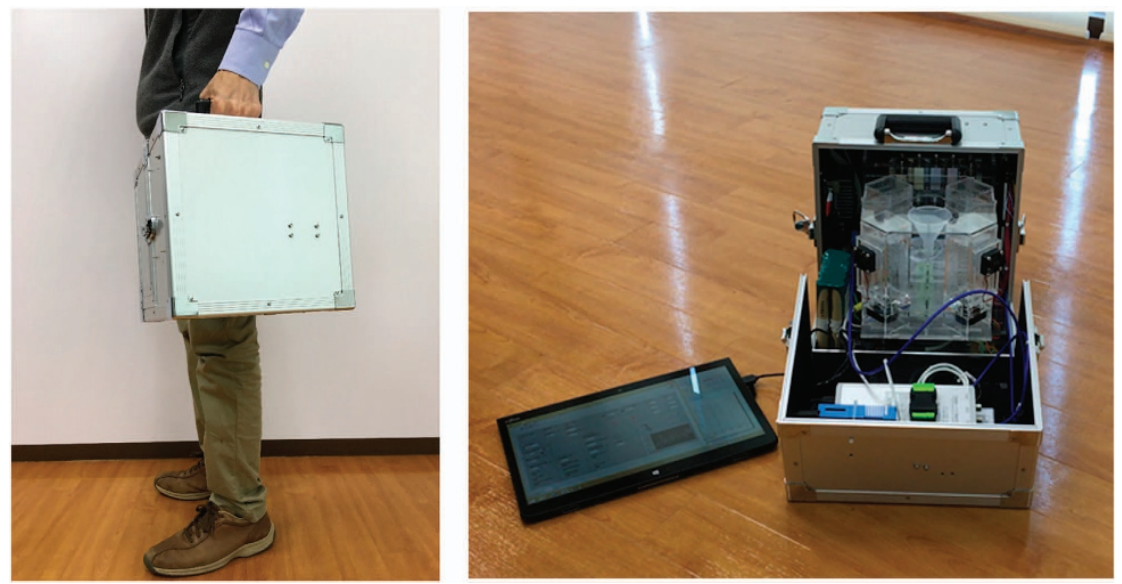

Figure 6 Prototype of the integrated automated portable device. All parts of the device are assembled in a compact $300 \mathrm{~mm} \times 300 \mathrm{~mm} \times 300 \mathrm{~mm}$ and $12.8 \mathrm{~kg}$ container. The device runs with a $24-\mathrm{V}$ battery power source and is connected to a tablet screen.

Blue bars indicate the results of the segment-flow PCRs using the aerosol sampler. These fluorescence intensities after the segmentflow PCRs do not only relate to the amount of the sprayed BS spores; these were also reasonable values between the results of both controls. While the BS spore solution was diluted 100 times (equal to $8 \mathrm{cfu} \mathrm{LL}^{-1}$ ), the fluorescence was successfully increased compared with the NTC. Here, chemical agents should be used with the assumption that the lethal dose in air is for $1 \mathrm{~min}$ of inhalation since the toxicity effect will appear immediately. Alternately, a pathogen agent should have the assumption that the lethal dose in air for $1 \mathrm{~h}$ of inhalation, because the infection of a bacterium pathogen or virus takes propagation time and has the greater possibility of treatment. The infection dose of anthrax spores is $8000-50000$ spores $^{34,35}$. If a human inhales approximately $81 \mathrm{~min}^{-1}$, the lethal dose of anthrax is calculated to be 16000 spore $\mathrm{m}^{-3}$. If it is assumed that the infection can be medically treated with the administration of antibiotics after early discovery of the agent exposure, the safety margin of the lethal dose is increased by 10 times $^{35}$. Thus, 54000 spores should be collected and detected, as required for anthrax sensitivity, when air is collected for $338 \mathrm{Imin}^{-1}$ by our sampling device. A concentration of $8 \mathrm{cfu} \mathrm{LL}^{-1}$, which was successfully detected by our device, has 25000 spores and covered the required sensitivity for anthrax detection. Therefore, it is expected that the sensitivity of the developed system would be enough for detecting biological warfare similar to anthrax spores. In addition, for $800 \mathrm{cfu} \mathrm{mL}^{-1}$, the observed fluorescence intensity was $8.38 \pm 1.05$ with a C.V. value of $12.5 \%(n=3)$ that demonstrate the reliability of the measurement.

\section{CONCLUSION}

First responders, such as the police and fire fighters, greatly need a multibiosensing mobile system with minimum manipulation for on-site detection for rapid risk assessment and analysis of the possible presence of $\mathrm{BC}$ agents in the event site. In this report, we have developed an autonomous air sampling and detection system, which integrates aerosol sampling and biosensing technologies. The designed collection system $\left(3381 \mathrm{~min}^{-1}\right)$ was able to collect the target BC agents. More importantly, concentrations lower than the mean lethal dose $\left(\mathrm{LD}_{50}\right)$ were successfully detected for nerve gases (sarin and VX) by electrochemical measurement, toxic proteins (BTX/A/Hc and ricin) by LSPR measurement, and pathogens (anthrax simulant) by chip PCR. An operation time of only $5-15 \mathrm{~min}$ is needed for the collection and detection. The detection of each BC was done separately, but it is envisioned that the device is capable of simultaneous detection. Due to technical limitations in preparing a model gas containing all the BC targets, this was not demonstrated. More importantly, neither cross-contamination nor false signals was found for BC targets present in unintended sensing device (Supplementary Figure S7 and Supplementary Table S2), which promotes the reliability of the obtained measurements. Finally, to realize our system concept, we produced a compact and batteryoperated device having all of the units integrated into one system (Figure 6). However, it should be noted that the device is intended for first responders' utility, and it is expected that the findings will be validated again in testing labs after the necessary actions are made based on the risk assessment of on-site events. Still, with the features of the developed device, it is greatly expected to contribute to maintaining a safe and healthy society.

\section{ACKNOWLEDGEMENTS}

This work was financially supported by 'a R\&D Program for Implementation of AntiCrime and Anti-Terrorism Technologies for a Safe and Secure Society,' Funds for Integrated Promotion of Social System Reform and Research and Development of the Ministry of Education, Culture, Sports, Science and Technology (MEXT) of Japan, and a Grant-in-Aid for Scientific Research from MEXT.

\section{COMPETING INTERESTS}

The authors declare no conflict of interest.

\section{REFERENCES}

1 Danzig R, Sageman M, Leighton T et al. Aum shinrikyo: Insights into How Terrorists Develop Biological and Chemical Weapons. Center for a New American Security: Washington DC, USA, 2011.

2 Seto Y, Tsunoda N, Kataoka M et al. Toxicological analysis of victims blood and crime scene evidence samples in the sarin gas attack caused by the Aum Shinrikyo Cult. ACS Symposium Series Natural and Selected Synthetic Toxins; 1999: 318-332.

32015 Report on adherence to and compliance with arms control, nonproliferation, and disarmament agreements and commitments. US Department of State, 5 June 2015. www.state.gov/t/avc/rls/rpt/2015/243224.htm.

$4 \mathrm{Kim}$ K, Tsay OG, Atwood DA et al. Destruction and detection of chemical warfare agents. Chemical Reviews 2011; 111: 5345-5403.

5 Jang YJ, Kim K, Tsay OG et al. Update 1 of: destruction and detection of chemical warfare agents. Chemical Reviews 2015; 115: PR1-PR76.

6 Ohsawa I, Kanamori-Kataoka M, Tsuge K et al. Determination of thiodiglycol, a mustard gas hydrolysis product by gas chromatography-mass spectrometry after tert-butyldimethylsilylation. Journal of Chromatography A 2004; 1061: 235-241. 
7 Seto $\mathrm{Y}$, Kanamori-Kataoka M, Tsuge K. Mass spectrometric technologies for countering chemical and biological terrorism incidents. Journal-Mass Spectrometry Society of Japan (in Japanese) 2008; 56: 91.

8 McBride MT, Masquelier D, Hindson BJ et al. Autonomous detection of aerosolized bacillus anthracis and Yersinia p estis. Analytical Chemistry 2003; 75: 5293-5299.

9 Hindson BJ, McBride MT, Makarewicz AJ et al. Autonomous detection of aerosolized biological agents by multiplexed immunoassay with polymerase chain reaction confirmation. Analytical Chemistry 2005; 77: 284-289.

10 Zhang X, Young MA, Lyandres $\mathrm{O}$ et al. Rapid detection of an anthrax biomarker by surface-enhanced Raman spectroscopy. Journal of the American Chemical Society 2005; 127: 4484-4489.

11 Peruski AH, Peruski LF. Immunological methods for detection and identification of infectious disease and biological warfare agents. Clinical and Diagnostic Laboratory Immunology 2003; 10: 506-513.

12 Abu-Qare AW, Abou-Donia MB. Sarin: Health effects, metabolism, and methods of analysis. Food and Chemical Toxicology 2002; 40: 1327-1333.

13 van der Schans MJ, Lander BJ, van derWiel $\mathrm{H}$ et al. Toxicokinetics of the nerve agent $( \pm)-V X$ in anesthetized and atropinized hairless guinea pigs and marmosets after intravenous and percutaneous administration. Toxicology and Applied Pharmacology 2003; 191: 48-62.

14 Leon-Gonzalez ME, Townshend A. Flow-injection determination of paraoxon by inhibition of immobilized acetylcholinesterase. Analytica Chimica Acta 1990; 236: 267-272.

15 Guilbault GG, Kramer DN. Fluorometric system employing immobilized cholinesterase for assaying anticholinesterase compounds. Analytical Chemistry 1965; 37: $1675-1680$.

16 Halámek J, Makower A, Knösche K et al. Piezoelectric affinity sensors for cocaine and cholinesterase inhibitors. Talanta 2005; 65: 337-342.

17 Liu B, Yang YH, Wu Z-Y et al. A potentiometric acetylcholinesterase biosensor based on plasma-polymerized film. Sensors and Actuators B: Chemical 2005; 104: 186-190.

18 Pohanka M, Adam V, Kizek R. An acetylcholinesterase-based chronoamperometric biosensor for fast and reliable assay of nerve agents. Sensors 2013; 13: 11498-11506.

19 Yamanaka K, Saito M, Kondoh K et al. Rapid detection for primary screening of influenza A virus: Microfluidic RT-PCR chip and electrochemical DNA sensor. Analyst 2011; 136: 2064.

20 Nagatani N, Yamanaka K, Saito M et al. Semi-real time electrochemical monitoring for influenza virus RNA by reverse transcription loop-mediated isothermal amplification using a USB powered portable potentiostat. Analyst 2011; 136: 5143.

21 Yamanaka K, Sekine S, Uenoyama T et al. Quantitative detection for porphyromonas gingivalis in Tooth Pocket and saliva by portable electrochemical DNA sensor linked with PCR. Electroanalysis 2014; 26: 2686-2692.

22 Nagatsuka T, Uzawa H, Sato $\mathrm{K}$ et al. Localized surface plasmon resonance detection of biological toxins using cell surface oligosaccharides on glyco chips. ACS applied materials \& interfaces 2013; 5: 4173-4180.
23 Fuchiwaki $Y$, Nagai $H$, Saito $M$ et al. Ultra-rapid flow-through polymerase chain reaction microfluidics using vapor pressure. Biosens Bioelectron 2011; 27: 88-94.

24 Chemical Management Directory. Ministry of Economy, Trade, and Industry (METI), Japan. http://www.meti.go.jp/policy/chemical_management/cwc/index. html.

25 Hoffmann AC, Stein LE. Gas Cyclones and Swirl Tubes: Principles, Design, and Operation. Springer Science \& Business Media: New York, USA. 2013.

26 Del Carlo M, Mascini M, Pepe A et al. Screening of food samples for carbamate and organophosphate pesticides using an electrochemical bioassay. Food Chemistry 2004; 84: 651-656.

27 Bucur B, Fournier D, Danet A et al. Biosensors based on highly sensitive acetylcholinesterases for enhanced carbamate insecticides detection. Analytica Chimica Acta 2006; 562: 115-121.

28 Naoki N, Akihiro T, Hossain MA et al. Rapid and sensitive visual detection of residual pesticides in food using acetylcholinesterase-based disposable membrane chips. Food Control 2007; 18: 914-920.

29 Bajgar J. Complex view on poisoning with nerve agents and organophosphates. Acta Medica (Hradec Kralove) 2005; 48: 3-21.

30 Shi M, Xu J, Zhang S et al. A mediator-free screen-printed amperometric biosensor for screening of organophosphorus pesticides with flow-injection analysis (FIA) system. Talanta 2016; 68: 1089-1095.

31 Liu G, Lin Y. Biosensor based on self-assembling acetylcholinesterase on carbon nanotubes for flow injection/amperometric detection of organophosphate pesticides and nerve agents. Analytical Chemistry 2006; 78: 835-843.

32 Ivarsson U, Nilsson H, Santesson J. A FOA Briefing Book on Chemical Weapons: Threat, Effects, and Protection. Forsvarets Forskningsanstalt, Ursvik and Sorunda, Sweden. 1992.

33 Arnon SS, Schechter R, Inglesby TV et al. Botulinum toxin as a biological weapon: Medical and public health management. Jama 2001; 285: 1059-1070.

34 Franz DR. Clinical recognition and management of patients exposed to biological warfare agents. Jama 1997; 278: 399-411.

35 Seto Y. On-site detection method for warfare agents. Bunseki Kagaku 2006; 55 891-906.

This work is licensed under a Creative Commons Attribution 4.0 International License. The images or other third party material in this article are included in the article's Creative Commons license, unless indicated otherwise in the credit line; if the material is not included under the Creative Commons license, users will need to obtain permission from the license holder to reproduce the material. To view a copy of this license, visit http://creativecommons.org/licenses/ by/4.0/

(c) The Author(s) 2018

Supplementary Information for this article can be found on the Microsystems \& Nanoengineering website (http://www.nature.com/ micronano) 